\title{
Enhanced IHMAC with Dead Line Aware Packet Scheduling Scheme for Wireless Sensor Networks
}

\author{
Y. Sravya Lakshmi, \\ VBIT, Hyderabad \\ India
}

\author{
Bhaskar Gugulothu, \\ VBIT, Hyderabad, \\ India
}

\begin{abstract}
Wireless detector networks have become very democratic in Holocene years. For detector network, S-MAC and T-MAC are used to improve the energy benefits by applying sleep control. Our ultimate aim is to achieve high power benefits under extended range of traffic and also promises shorter delay for this type of emergency and latency-sensitive messages and removed collision. In this project, we present the light weight Intelligent Hybrid MAC, a newer low power with QoS promising medium access control protocol for wireless detector networks. Our proposed Enhanced ACTIVITY CONTROL (Enhanced-IHMAC) is combines TDMA and CSMA with buffering technique. There are many applications of wireless detector network where it is really wanted to ensure the priority services for the emergency information. And also we have not used synchronization messages unlike our base model. Our proposed ACTIVITY CONTROL (EnhancedIHMAC) protocol guarantees shorter latency for this type of emergency and latency-sensitive messages with low overhead and emergency scheduling.
\end{abstract}

Key words: WDN, power saving, sleep mode, priority, MAC

\section{INTRODUCTION}

A wireless detector network (WDN) consists of detector devices capable of collecting information from the place and sharing the information with each other via wireless devices. The collected data will be delivered to one or more destinations, very often through multi-hop information sharing. The detector devices are very often expected to operate with power units and are often deployed to not-easily-accessible or remote place, sometimes in prominent numbers. It can be unmanageable or impossible to replace the power units of the detector devices. On the other hand, the destination is very often rich in power. Since the detector power is the most precious resource in the WDN, effective utilization of the energy to prolong the lifetime of network has been the main focus of the research on the WDN.

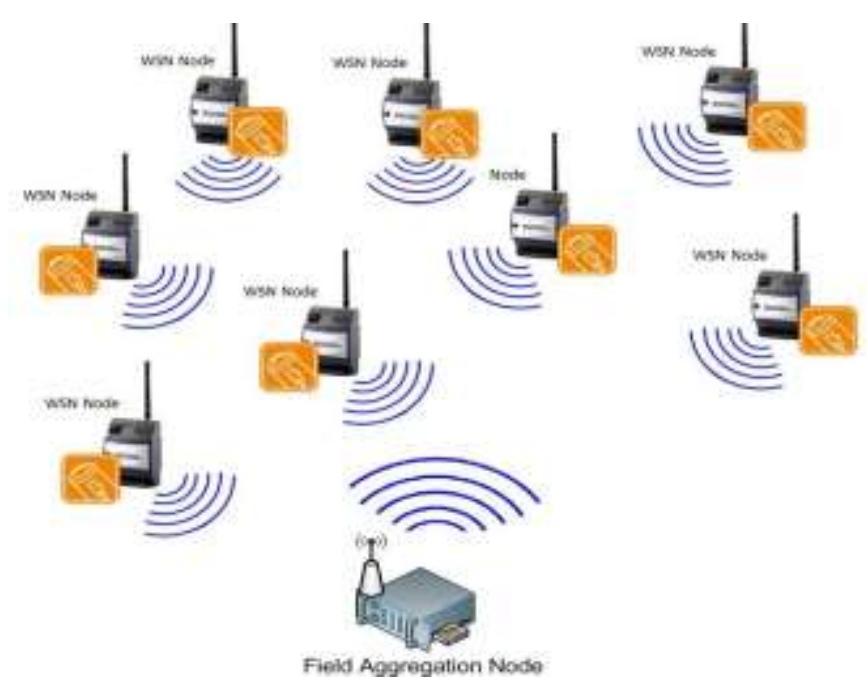

Fig.1 wireless detector network

The information sharing's in the WDN has the many-toone mode in that data from a prominent number of detector devices tend to be concentrated into a few destinations. Since multi-hop routing is very often wanted for distant detector devices from the destinations to save power, the devices near a destination can be burdened with relaying a prominent amount of traffic from other devices. Communication in wireless detector networks will, like most network communication, be divided into many layers. one in all those is that the Medium Access control (MAC) layer. This layer is delineated by a Mac protocol, that tries to make sure that no two nodes are officious with every other's transmissions, 
and deals with things once they do. Wireless detector networks have a further aspect: as detector nodes are typically batteryoperated, energy consumption is incredibly necessary. The radio on a detector node is sometimes the part that uses most energy. Not solely transmission prices energy; receiving, or just scanning the ether for communication, will expand to half the maximum amount, reckoning on the sort of radio [8]. Whereas ancient mackintosh protocols are designed to maximize packet output, minimize latency and supply fairness, protocol style for wireless detector networks focuses on minimizing energy consumption. the appliance determines the wants for the (modest) minimum output and most latency. Fairness is sometimes not a problem, since the nodes in a very wireless detector network are generally a part of one application and work along for a standard purpose

\section{RELATED WORK}

This paper [1] presents RAP, new real-time information sharing architecture for prominent-scale detector networks. In some of previous work the Authors proposed Velocity Monotonic Scheduling. VMS assigns the priority to a message bundle based on its requested speed. A message bundle with a higher requested speed is assigned a higher priority. VMS improves the number of messages that meet their deadlines because it assigns the "right" priorities to messages based on their emergency on the current node. But there is no detail for message bundle receiving distance in that paper. And in this paper, when the queue is filled, most priority incoming messages overwrite the lower priority messages at ones. It may trouble to be the low priority information.

In paper [2], Authors proposed an Adjustive Staggered SLEEP Protocol for effective energy management in wireless detector networks targeted to periodic data collection. This algorithm lively adjusts the sleep schedules of devices to match the network needs, even in time-changing operating conditions. It utilizes the CSMA scheme for process the info, but it may be not efficient in fixed WDN network and there is no detail to data handling. In this paper [2], Authors presented how to place detectors by use of a minimal number to maximize the coverage area when the information sharing radius of the $\mathrm{SN}$ is not less than the detecting radius, which results in the application of regular topology to WDNS placement.

In this paper Authors discussed the details of detector placement. Due to optimal coverage detector placement, it reduces the no of detectors usage and also step-ups the lifetime of detectors. But till lifetime of detector need to step-up. In paper [3], Authors proposed a grouping method with coverage and power aware TDMA scheduling method. And the group creation is done by the base station according to the current residual power, and the coverage area of group Member is reduced to avoid the congestion and power management. In that paper also there is no discussion on the real time and non-real time message bundle transmission. Authors developed scheme by deploying the network with different in size and fixed grids while taking into account the arbitrary shaped area sensed by the detector devices.
In paper [4], Authors considers the different initial power level of detectors, and placing that detector according to that power range. So power reduction was for fended. But measuring different initial power level and placing the device according to that power level is unmanageable in real time.

In paper [5] Authors presented a group based routing algorithm. One of main goal is to design the power efficient routing protocol. This algorithm makes the best use of device with low number of group leader know as super device. Here Authors divided the full area in equal zones and the center area of the area is used to select for special device. Every area is considered separately and the zone may be or not divided further that's depending upon the tightness of devices in that zone and capability of the special device. In this paper Authors considered, group leader changes when the group leader is failed. It may be the trouble to detecting in that area.

\subsection{Existing system \& demerits}

The Existing Z-MAC has the initial phase. In initial phase there are neighbor node identification, timing assignment, neighbor message exchange and global time synchronization steps has been done. Q-MAC method gives QoS by differentiating network services based on priority values. The priority values reflect the emergency of messages sectioning from different detector devices.

\section{PROPOSED SYSTEM}

The ACTIVITY CONTROL (Enhanced-IHMAC) does it by using the strength of carrier sensing and time division approach with intelligence. The innovative idea behind the ACTIVITY CONTROL (Enhanced-IHMAC) is that it uses both the broadcast scheduling and link scheduling. ACTIVITY CONTROL (Enhanced-IHMAC) classifies messages according to their emergencies and stored the messages into the appropriate queue. The source device knows the degree of importance of the sensed information and accordingly the application layer assigns the priority.

\subsection{Techniques}

○ Route Discovery

(]) Timer

$4 \quad$ Sectioning Message Generator

(1) Route establishment

○ Virtual Grouping

(] Allocation of Sections

- Data Transmission/Buffering

○ Power Saving 


\subsubsection{Route Discovery}

Here we are going to enable the timer to send sectioning message in specific intervals. Sectioning message is to know about the neighbor devices sections, based on the reply; device will store the neighbor table information. The Request message will flood to the each device in the network to update the information of base station availability.

\subsubsection{Power Saving}

The ACTIVITY CONTROL uses Request-To-Send, ClearTo-send messages to handshake with the neighbor device. And this method is used to adjust the transmitting power to the minimum level necessary to reach the intended neighbor. Thus, the ACTIVITY CONTROL (Enhanced-IHMAC) reduces power loss by suitably changing the transmit power. And this handshaking method used to synchronize the sleep scheduling also.

\subsubsection{Data Transmission}

According to the energy saving method and in the allocated sections based on handshaking method, detectors are going to transmit the data and similarly they will change to sleep mode when there is no transmission.

In our proposed system, we have implemented ACTIVITY CONTROL with reduced over leader model. As like as in TDMA method, we also divided time into time sections, but unlike TDMA method each device can use the other time section when the time section is not in use. To check whether time section is 'not in use' or use, we have connected CSMA/CA method. In our project, first we divided time into time sections and then further we divided time section into further three sections (priority section, own section, other section). If device has any priority data then the device can transfer the data at beginning of time section which may be own or others. If device not has any priority data then it will check for section is for me or not. If section is own section then it can send data in second section of main section.

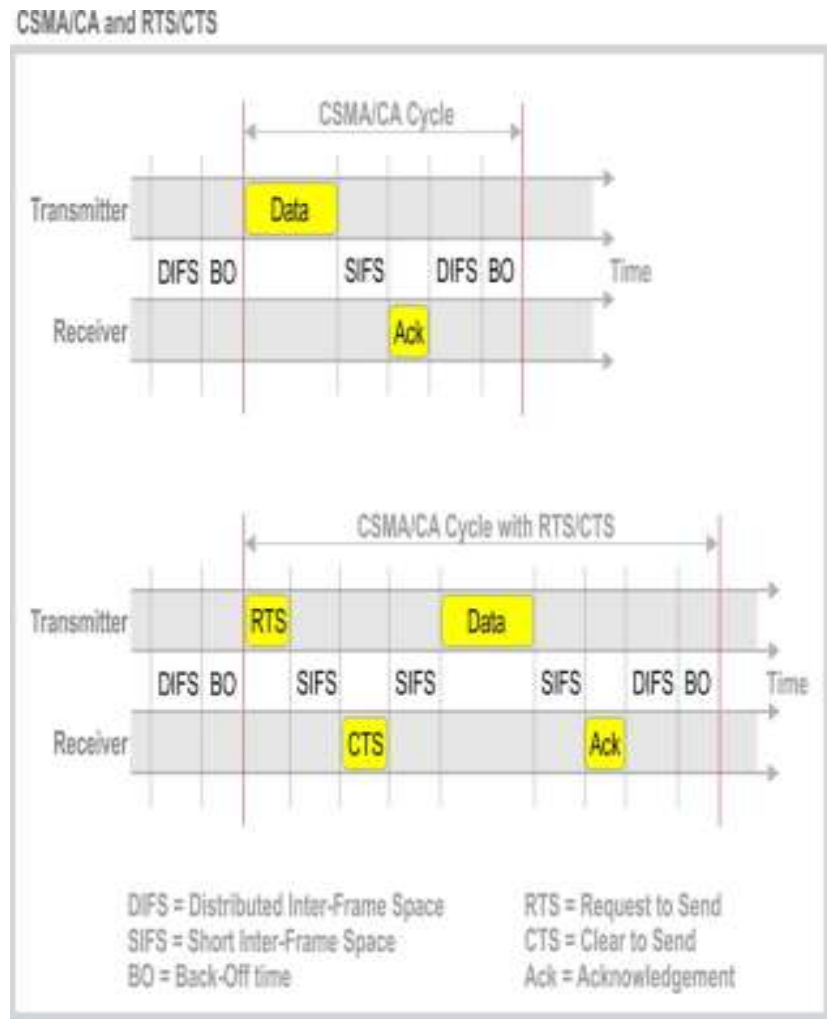

Fig.2 CSMA/CA method

If section is others section then it will wait for third section in main section with small random interval if device not detecting information sharing then device can transfer the data in that section. Fig.7 shows the result of data transmission through other section

\subsection{4) Priority message bundle scheduling}

Among large number of network design problems, such as routing protocols and data collection, that reduce sensor energy loss and data transmission delay, message bundle scheduling (interchangeably use as task scheduling) at sensor devices is highly important since it ensures delivery of different types of messages based on their priority and fairness with a lesser delay. Indeed, most existing Wireless Detector Network use FCFS schedulers that process messages in the order of their receiving time and, thus, need a large amount of time to be delivered to a relevant base station. However, to be useful, detected information has to reach the BS within a certain period or before the deadline. Additionally, emergency info should be delivered to base station with the lesser possible end-to-end latency. Hence, intermediate devices require changing the delivery order of data messages in their ready queue based on their importance and delivery priority. In our enhanced work, we have considered the buffering problem; the message bundle may chances to loss due to scheduling at a time. So in our enhancement work the device can remember the previous scheduling message bundle information. We are 
concentrating on reducing the overhead and avoiding the packet loss due to inefficient packet scheduling. In our base work, the researcher used the synch packet to make synchronization. Once the node gets the slot to transfer the data then it has to synchronize with neighbor to deliver the data to next node. To synchronize, the sender node broadcasts the synch message to neighbors with the name of indented receiver. If the neighbor received then it has to check whether own id is present in found in that or not. If own id found then it can know that it should be in active or else it can go to in sleep. In our enhancement work, we have proposed the RTS synchronization. If any node received the RTS/CTS signal then it has to check whether own id is found or not, if own id is not found then the device has to go to sleep otherwise node will be in active. So we can reduce unnecessary overhead in the network. RTS/CTS message is enough to synchronize the network.

In our base work, there are no details to schedule the multiple priority packets at one node. In case, the node generated one packet with priority and received the one priority packet at same time then the node has to schedule both at same time. If both packets are scheduled at same time then any one of the message bundle will be successfully scheduled and another one will be deleted. In our enhanced work we have considered this problem. So in our enhancement, the node checks the scheduling info whether any packet is already scheduled or not, if priority packet is already scheduled then another priority packet will be added into the queue. So that message bundle can send in next time slot without loss. We can improve the packet delivery by queuing technique.

\section{2) Algorithm}

\subsection{1) Algorithm:}

Inputs: $\mathrm{N}$-number of sensor nodes, 1-base station node. At beginning pre-defined sensor will generate slot req, after some predefined duration base station will share origination message.

1) Sensor "i" sends the slot req to immediate neighbors $(1,2 \ldots \mathrm{m}) \quad(\mathrm{m}<15)$ and set the timer to confirm the slot (confirmation timer)

2) If slot req received in any sensor

a. Checks the waiting list and confirmation list

i. If No slot is matching with requested slot

1. Add the requested slot into waiting list with node id, time and hop cont

ii. If requested slot already available in confirmed list

1. Generates the rejection message with

a. Node id

b. Confirmed slot info

iii. If request not found in confirmed list but available in waiting list

1. Check the time of slot req generation a. If new slot_req_time <already available pending req_time

i. Add new req in waiting list

ii. Reject already available pending slot_req

b. If new slot_req_time >already available pending req_time

i. Reject new req, and send rejection mesg

c. If new slot_req_time >already available pending req_time

i. Reject both and send rejection mesg

3) If rejection messg received in any sensor node

a. If rej_mesg is for me

i. Then reset the own slot

ii. Then send cancelation message to all neighbor node

iii. And checks confirmed slot info from pkt and updates the confirmed list

b. If not for me

i. checks confirmed slot info from pkt and updates the confirmed list

4) if cancelation message received then

a. check the canceller info in waiting list

i. if found

1. delete the info

ii. else

1. ignore

5) if in any sensor confirmation timer is expire after sending the slot req

a. checks it own slot info in own waiting list (slot requested sensor)

i. if info found with requested slot

1. confirm the slot for own use

2. send the confirmation message to all neighbors

ii. if info not found

1. set the timer to re-allocate the slot

6) if confirmation message received

a. update the confirm list

b. checks the own confirm list for own slot

i. if node has own lot

1. ignore the pkt

ii. if node not has any slot for own use then

1. set the limited random time to generate slot req

\subsection{2) Data transfer}

If synchronization is completed

I) if data generated

a. set the mode (priority or non-priority)

b. checks the packet priority

i. if priority- packet

1. then set the packet sending in next section starting

ii. if non-priority- packet

1. then add into buffer 
International Journal of Computer Applications Technology and Research

Volume 4- Issue 11, 871 - 877, 2015, ISSN: 2319-8656

II) if data recv

a. continue the same process as $I-b$

III) if current time is for next section

a. count the current section number

b. if section number matching with own section

i. set the timer for own section access

c. if section number is others

i. set the timer for other section access

d. schedule next section

IV) if current time to access own section

a. check the mac

i. if not in use

$$
\text { 1. send the data }
$$

ii. if not

1. re-buffer the packet

$\mathrm{V})$ if current time to access other section (continue the process of $(I V-a))$

VI) if current time is to emergency packet

a. Go to buffer scheduling algorithm

\subsection{3) Buffer scheduling algorithm:}

Each node has to use CSMA/CA technique to transfer the data in allocated TDMA slot. Let consider $f$ forwarder of data and $n$ is receiver

1) If slot initiated

a. If packet. data available in $\mathrm{f}$

i. If can use Sub slot

1. Generate RTS packet

2. $(\mathrm{f}, \mathrm{n}) \cup$ packet. addrs

3. broadcast RTS

ii. Else

1. Wait for next sub slot

b. Else

i. Switch to idle mode

2) If packet. RTSreceived in $n$

a. If $n \exists$ packet. addrs

i. Switch to Active state

ii. Send CTS $\ni(\mathrm{f}, \mathrm{n})$

b. Else

i. Set NAV

ii. Switch to sleep till next slot initiation

3) If packet. CTS recv in $n$

a. If $\mathrm{n} \exists$ packet. addrs

i. Switch to Active state

ii. Send data

b. Else
i. Set NAV
ii. Switch to sleep till next slot initiation

4) If packet. data recv in $n$

a. If packet. data emergency

i. Get interval nslot ii. If $\exists$ schedule

iii. Else

1. En-queue packet

1. schedule packet

\subsection{4) power saving algorithm}

(I) Power control technique

A) set the maximum transmission power

B) while sending RTS/CTS set maximum power as tx power

C) if CTS pkt is received

a. checks the recv power info

b. set new tx power $P_{t x}=\frac{P_{\max }}{P_{\text {rec }}} * P_{r t h} * C$

(II) Sleep

a. If node ready to send data

i. Send synch message with $(\operatorname{addrs}(\mathrm{F}, \mathrm{N}))$

b. If Synch recv

i. If node is not in synch

1. Make node to sleep

\section{RESULT ANALYSIS}

We have tested our proposed system with the Network Simulator 2 tool. Our proposed system the delay is less than all other slandered systems (see fig. 3 )

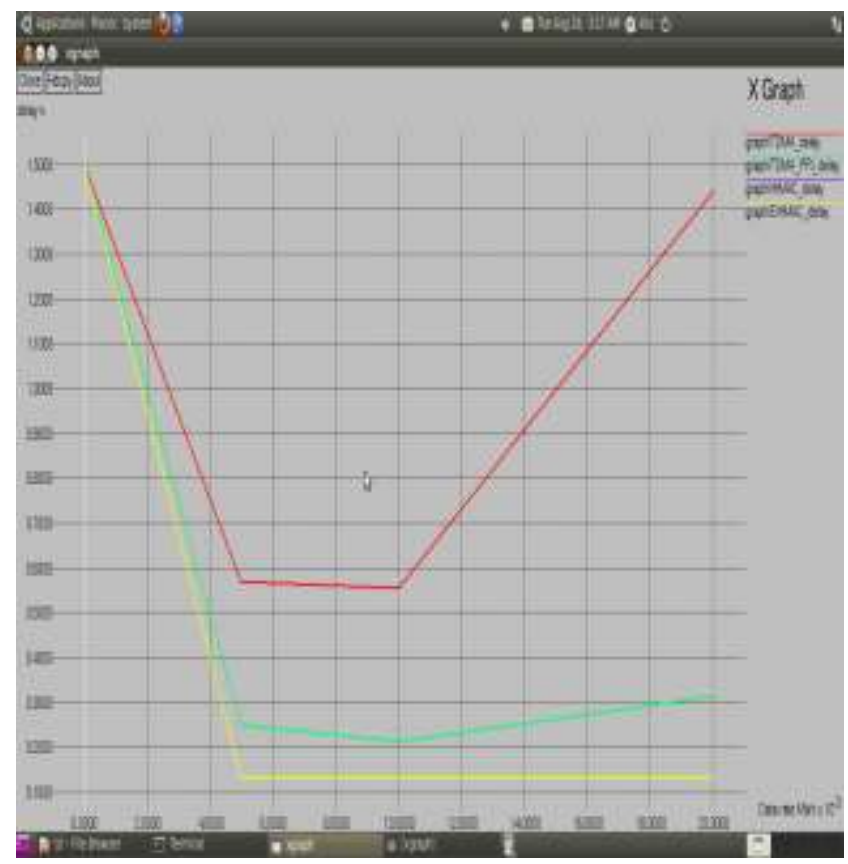

Fig.3 delay comparison

Our proposed system provides less energy loss compare than all other standard methods (see fig.4) 


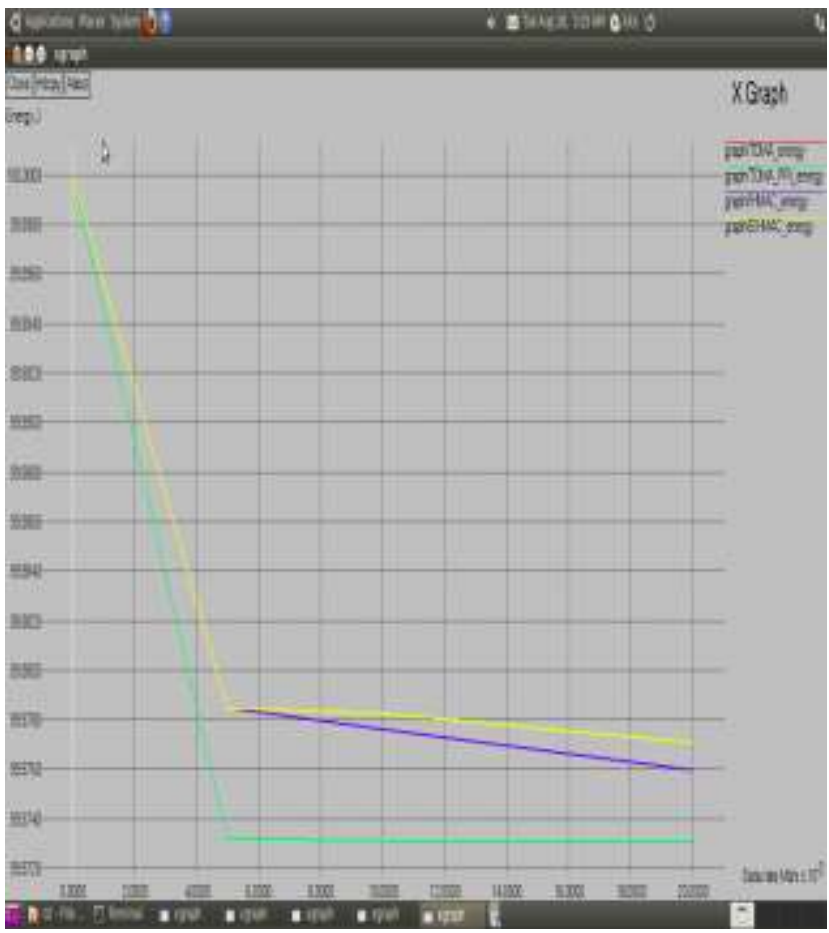

Fig.4 Energy comparison

In our enhanced method, we have not used the synch packets so our enhancement method provides less overhead compare than all other standard methods (see fig.5)

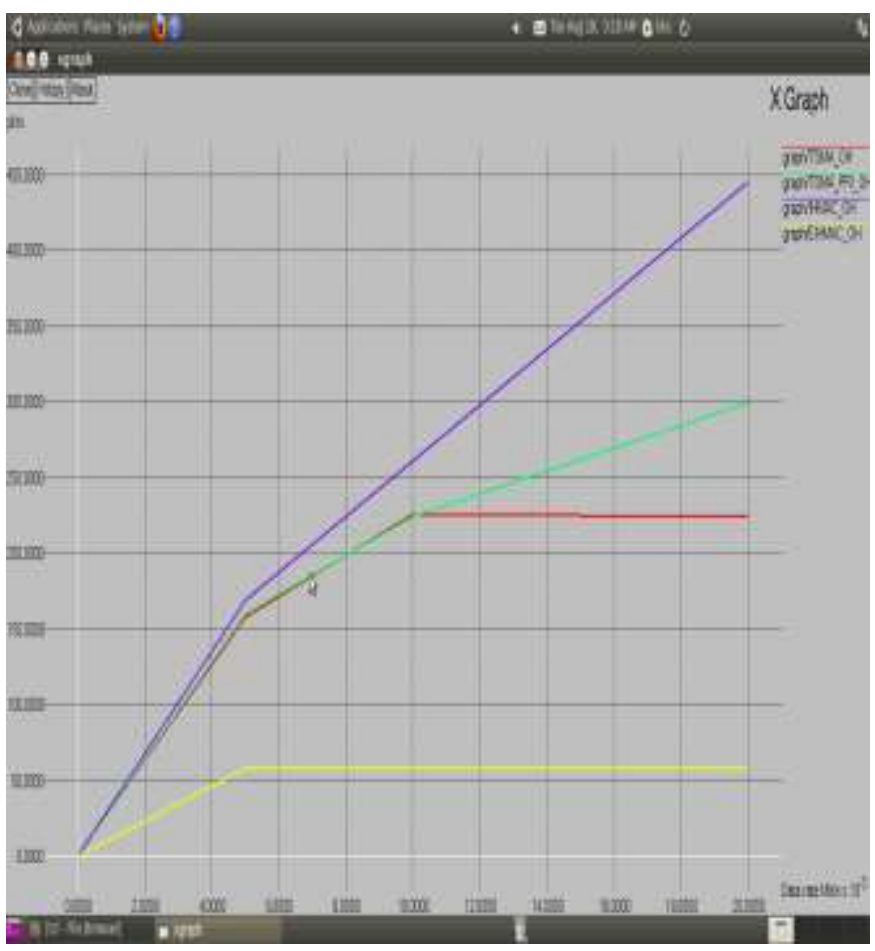

Fig.5 overhead comparison
Our proposed system provides higher stable packet delivery compare than all other standard methods (see fig.6)

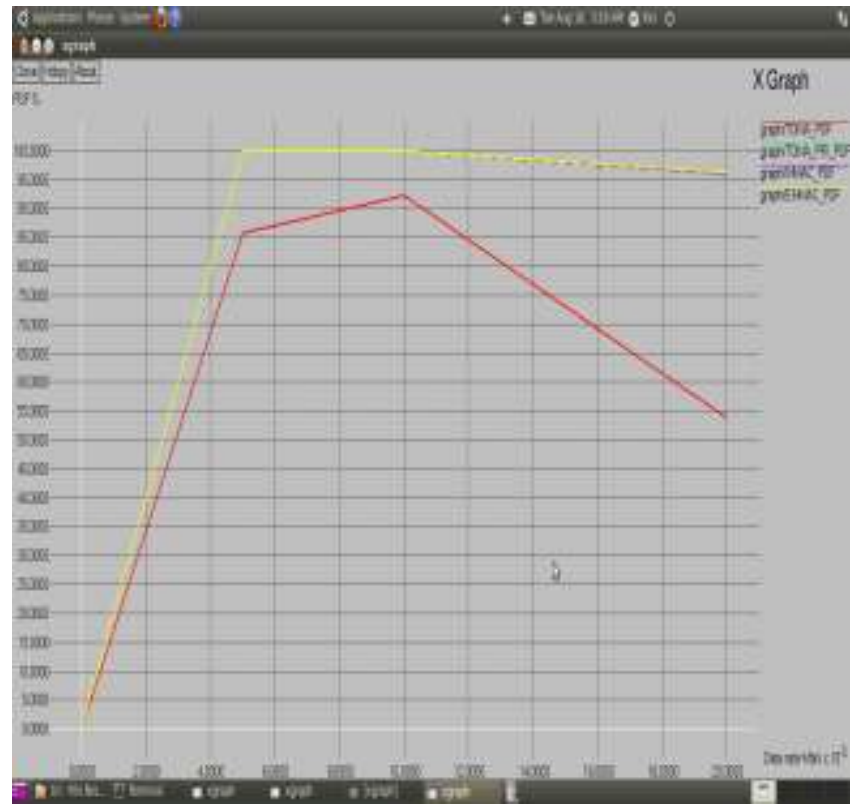

Fig.6 PDF comparison

\section{CONCLUSION}

In this paper, we have presented the ACTIVITY CONTROL (Enhanced-IHMAC), a newer low energy with QoS promising medium access control protocol for wireless detector networks. Our proposed ACTIVITY CONTROL is combines Time division and carrier sensing techniques. There are many applications of wireless detector network really wanted to ensure the priority services for the emergency data. Our proposed ACTIVITY CONTROL protocol promises the shorter latency for this type of emergency and latency-sensitive messages. And we have proved our method is lightweight power saving method. In our future work, we will study the details of mobile detector devices.

\section{REFERENCES}

1) Chenyang Lu, Brian M. Blum, Tarek F. Abdelzaher, John A. Stankovic, Tian He, "RAP: A Real-Time Information sharing Architecture for Prominent-Scale Wireless Detector Networks", 2002.

2) Giuseppe Anastasi, Marco Conti, Mario Di Francesco, "Extending the Lifetime of Wireless Detector Networks through Adjustive Sleep", 2007 
International Journal of Computer Applications Technology and Research

Volume 4- Issue 11, 871 - 877, 2015, ISSN: 2319-8656

3) "Maximizing Networking Lifetime in Wireless Detector Networks with Regular Topologies", Hui Tian, Hong Shen and Matthew Roughan.

4) Ms.Namrata S. Baghele, "An Adjustive Power Aware Device Scheduling Scheme In Grouping for Wireless Detector Networks", 2012

5) “A Strategic Placement and Group-Leaderer Selection for Wireless Detector Networks", Tarandeep Kaur, and Jinsuk Baek, Member, IEEE.

6) Ashim Kumar Ghosh1, Anupam Kumar Bairagi2, Dr. M. Abul Kashem3, Md. Rezwan-ul-Islam1, A J M Asraf Uddin1 "Power Efficient Zone Division Multihop Hierarchical Grouping
Algorithm for Load Balancing in Wireless Detector Network.", 2011.

7) "Power-Efficient Group Formation for Prominent Detector Networks using a Minimum Separation Distance”, Ewa Hansen, Jonas Neander, Mikael Nolin and Mats Björkman.

8) Sinem Coleri Ergen and Pravin Varaiya, "TDMA Scheduling Algorithms for Wireless Detector Networks”, 2009

9) Renjie Huang, Wen-Zhan Song, Mingsen $\mathrm{Xu}, \mathrm{Behrooz}$ Shirazi, Guoliang Xing, "Localized QoS-Aware MAC Protocol for Real-time Dynamic Detector Networks"

10) Y. Liu, I. Elhanany, and H. Qi, "An power-efficient QoS-aware media access control protocol for wireless detector networks," Nov. 2005. 